\title{
High glucose, unsaturated and saturated fatty acids differentially regulate expression of ATP-binding cassette transporters $A B C A 1$ and $A B C G 1$ in human macrophages
}

\author{
Richard Mauerer ${ }^{1 *}$, Stefanie Ebert ${ }^{2 *}$ \\ and Thomas Langmann ${ }^{2,3}$ \\ ${ }^{1}$ Synlab Medical Care Service \\ Medical Care Centre Weiden \\ Zur Kesselschmiede 4 \\ 92637 Weiden, Germany \\ ${ }^{2}$ Institute of Human Genetics, University of Regensburg \\ Franz-Josef- Strauss-Allee 11, \\ 93042 Regensburg, Germany \\ ${ }^{3}$ Corresponding author: Tel, 49-941-944-5423; \\ Fax, 49-941-944-5402; \\ E-mail, thomas.langmann @klinik.uni-regensburg.de \\ *These authors contributed equally to this work. \\ DOI 10.3858/emm.2009.41.2.015
}

Accepted 3 November 2009

Abbreviations: CVD, cardiovascular disease; FFA, free fatty acids; LXR, liver-X-receptor; RXR, retinoic acid receptor; TLDA, TaqMan low-density arrays

\begin{abstract}
The ATP-binding cassette transporters ABCA1 and $A B C G 1$ are highly expressed in macrophage-derived foam cells and promote reverse cholesterol efflux via biogenesis of high-density lipoproteins. The aim of this study was to analyze the direct effects of bioactive factors related to the metabolic syndrome on macrophage transcript levels of all 47 human ABC transporters. Using in vitro M-CSF predifferentiated macrophages and TaqMan low density arrays we could show that linoleic acid, palmitic acid, and high glucose levels have a major impact on $A B C A 1$ and $A B C G 1$ expression but do not strongly affect most other human $A B C$ transporters. In Western blot experiments we demonstrate that ABCA1 and ABCG1 protein levels are synchronously suppressed by high glucose levels and the $\omega 6$-unsaturated fatty acid linoleic acid. We conclude that metabolites associated with the metabolic syndrome enhance the formation of atherosclerotic lesions by diminishing the reverse cholesterol transport function of $A B C A 1$ and ABCG1.
\end{abstract}

Keywords: ABCG1 protein, human; atherosclerosis;
ATP binding cassette transporter 1 ; gene expression profiling; macrophage; metabolic syndrome $\mathrm{X}$

\section{Introduction}

The metabolic syndrome is a concurrence of several metabolic risk factors (glucose intolerance, central obesity, elevated blood pressure and dyslipidemia) in one individual with increasing prevalence in Western populations. Importantly, patients with the metabolic syndrome have a significantly elevated risk of developing both type 2 diabetes and cardiovascular disease (CVD). One striking feature is atherogenic dyslipidemia, which is characterized by an elevation of triglycerides, free fatty acids (FFA) and proatherogenic small dense LDL particles, while anti-atherogenic HDL are decreased. In addition, this disordered lipid metabolism and high levels of FFA contribute to the development of insulin resistance, another important feature of the metabolic syndrome (Lee et al., 2006; Savage et al., 2007).

Atherosclerosis is histopathologically characterized by an accumulation of foam cells in the vessel wall. These foam cells are monocyte-derived macrophages accumulating large amounts of modified (e.g. oxidized) LDL. Under physiological conditions, macrophages have a high capacity for HDLdependent reverse cholesterol transport. The formation of mature HDL particles mainly depends on the proper function of two cooperating ATP-binding cassette $(A B C)$ transporters, $A B C A 1$ and $A B C G 1$ (Schmitz and Langmann, 2005). Thus, a high expression of these transporters counteracts the development of atherosclerotic plaques. Interestingly, diets enriched in polyunsaturated fatty acids, e.g. linoleic acid, have been shown to lower total cholesterol mainly at the expense of a decreased HDL-cholesterol concentration in monkeys and humans (Khosla and Hayes, 1992). In contrast, diets rich in saturated fatty acids, e.g. palmitic acid, had no such effect. Whether this is due to an altered ABCtransporter expression has not been explored yet.

The transcriptional regulation of $A B C A 1$ and ABCG1 by modified lipoproteins and oxysterols via the nuclear receptors liver-X-receptor (LXR) and retinoic acid receptor (RXR) has been studied 
Table 1. ATP-binding cassette transporter mRNA expression in monocyte-derived macrophages differentiated in the presence of M-CSF for 5 days ( $5 \mathrm{~d} \mathrm{Mac}$ ) or 4 day predifferentiated cells which were stimulated with insulin (Ins), palmitic acid (PA), linoleic acid (LA), 9cis-retinoic acid (RA), T0901317 (T0), and high-dose glucose (GIc) for further $24 \mathrm{~h}$.

\begin{tabular}{|c|c|c|c|c|c|c|c|}
\hline Gene & $5 \mathrm{~d}$ Mac & Ins & PA & LA & RA & T0 & Glc \\
\hline ABCA1 & •. & •・ & $\cdots$ & 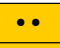 & $\cdots$ & $\cdots$ & • \\
\hline ABCA2 & $\cdots$ & $\cdots$ & $\bullet$ & $\cdots$ & $\cdots$ & $\cdots$ & $\cdots$ \\
\hline ABCA3 & $\cdots$ & $\cdot$ & $\cdot$ & $\cdot$ & $\cdot$ & $\cdot$ & $\cdot$ \\
\hline ABCA4 & - & - & - & - & - & - & - \\
\hline ABCA5 & $\cdots$ & $\cdots$ & $\cdots$ & $\cdots$ & $\cdots$ & $\cdots$ & $\cdots$ \\
\hline ABCA6 & $\cdots$ & $\cdots$ & $\cdots$ & $\cdots$ & $\cdots$ & $\cdots$ & $\cdots$ \\
\hline ABCA7 & - & $\cdot$ & $\cdot$ & $\cdot$ & $\cdots$ & $\cdots$ & $\cdot$ \\
\hline ABCA8 & - & - & - & - & - & - & - \\
\hline ABCA9 & $\cdot$ & - & $\cdot$ & $\cdot$ & $\cdot$ & $\cdot$ & $\cdot$ \\
\hline ABCA10 & - & - & - & - & - & - & - \\
\hline ABCA12 & - & - & - & - & - & - & - \\
\hline ABCA13 & - & - & - & - & - & - & - \\
\hline ABCB1 & - & - & - & $\cdot$ & $\cdots$ & $\cdots$ & - \\
\hline ABCB2 & $\cdots$ & $\cdots$ & $\cdots$ & $\cdots$ & $\cdots$ & $\cdots$ & $\cdots$ \\
\hline ABCB3 & $\bullet$ & $\bullet$ & 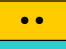 & $\bullet$ & $\bullet$ & $\bullet$ & 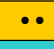 \\
\hline ABCB4 & $\cdot$ & - & $\cdot$ & • & $\cdot$ & $\cdot$ & • \\
\hline ABCB5 & - & - & - & - & - & - & \\
\hline ABCB6 & $\cdot$ & $\bullet$ & $\cdots$ & $\cdots$ & $\cdot$ & $\cdot$ & $\cdot$ \\
\hline ABCB7 & $\cdot$ & $\bullet$ & $\cdot$ & $\cdot$ & $\bullet$ & $\cdot$ & $\cdot$ \\
\hline ABCB8 & $\cdot$ & $\cdot$ & $\cdot$ & $\cdot$ & $\cdot$ & $\cdot$ & $\cdot$ \\
\hline ABCB9 & - & - & $\cdot$ & • & $\cdot$ & - & $\cdot$ \\
\hline ABCB10 & $\cdots$ & $\cdots$ & $\bullet$ & $\cdots$ & $\cdots$ & $\cdots$ & $\cdots$ \\
\hline ABCB11 & - & - & - & - & - & - & - \\
\hline ABCC1 & $\cdots$ & $\cdots$ & 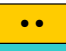 & $\cdots$ & $\cdots$ & $\cdots$ & $\cdots$ \\
\hline ABCC2 & - & $\cdot$ & • & $\cdot$ & $\cdots$ & $\cdots$ & • \\
\hline ABCC3 & $\cdots$ & $\cdots$ & $\cdots$ & $\cdots$ & $\cdots$ & $\cdots$ & $\cdots$ \\
\hline ABCC4 & $\cdots$ & $\cdots$ & $\cdots$ & $\cdots$ & $\cdots$ & $\cdots$ & $\cdots$ \\
\hline ABCC5 & $\cdots$ & $\cdots$ & $\cdots$ & $\cdots$ & $\cdots$ & $\cdots$ & $\cdots$ \\
\hline ABCC6 & - & $\cdot$ & $\cdot$ & $\cdot$ & $\cdot$ & $\cdot$ & $\cdot$ \\
\hline $\mathrm{ABCC} 7$ & - & - & - & - & - & - & - \\
\hline ABCC8 & - & - & - & - & - & - & - \\
\hline ABCC9 & - & - & - & - & - & - & - \\
\hline $\mathrm{ABCC} 10$ & 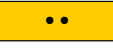 & $\cdots$ & $\cdots$ & $\cdots$ & $\cdots$ & $\cdots$ & $\cdots$ \\
\hline ABCC11 & - & - & - & - & - & - & - \\
\hline ABCD1 & $\bullet$ & $\cdots$ & •. & $\cdots$ & $\cdots$ & $\cdots$ & $\cdots$ \\
\hline ABCD2 & - & - & - & - & - & - & - \\
\hline ABCD3 & $\bullet$ & $\bullet$ & $\bullet$ & $\bullet$ & $\cdots$ & $\bullet$ & 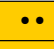 \\
\hline ABCD4 & $\bullet$ & $\bullet$ & $\bullet$ & $\bullet$ & $\cdots$ & $\cdots$ & $\bullet$ \\
\hline ABCE1 & $\bullet$ & 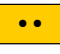 & $\bullet$ & $\bullet$ & $\bullet$ & 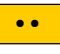 & 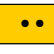 \\
\hline ABCF1 & $\cdot$ & $\cdot$ & $\cdot$ & $\bullet$ & $\cdot$ & $\cdot$ & $\cdot$ \\
\hline ABCF2 & $\cdots$ & $\cdots$ & $\cdots$ & 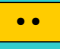 & $\cdots$ & $\cdots$ & . \\
\hline ABCF3 & $\cdot$ & $\cdot$ & $\cdot$ & - & $\cdot$ & - & - \\
\hline ABCG1 & $\cdots$ & $\cdots$ & $\cdots$ & $\cdots$ & $\cdots$ & $\cdots$ & $\cdot$ \\
\hline ABCG2 & - & $\cdot$ & $\cdot$ & $\cdot$ & $\cdot$ & • & $\cdot$ \\
\hline ABCG4 & - & - & - & - & - & - & - \\
\hline ABCG5 & - & - & - & - & - & - & - \\
\hline ABCG8 & - & - & - & - & - & - & - \\
\hline
\end{tabular}


extensively (Langmann et al., 1999; Klucken et al., 2000; Venkateswaran et al., 2000; Fu et al., 2001; Kennedy et al., 2001). In contrast, little information is available on the influence of plasma metabolites prevalent in the metabolic syndrome on ABCtransporter gene expression. Therefore, our aim was to study the influence of insulin, glucose, and saturated as well as unsaturated fatty acids on ABC-transporter gene expression in human monocyte-derived macrophages. For comparison, cells were cultured in the presence of the synthetic LXR/RXR-ligands T0901317 and 9-cis retinoic acid as known $A B C A 1 / G 1$ transcriptional regulators.

A

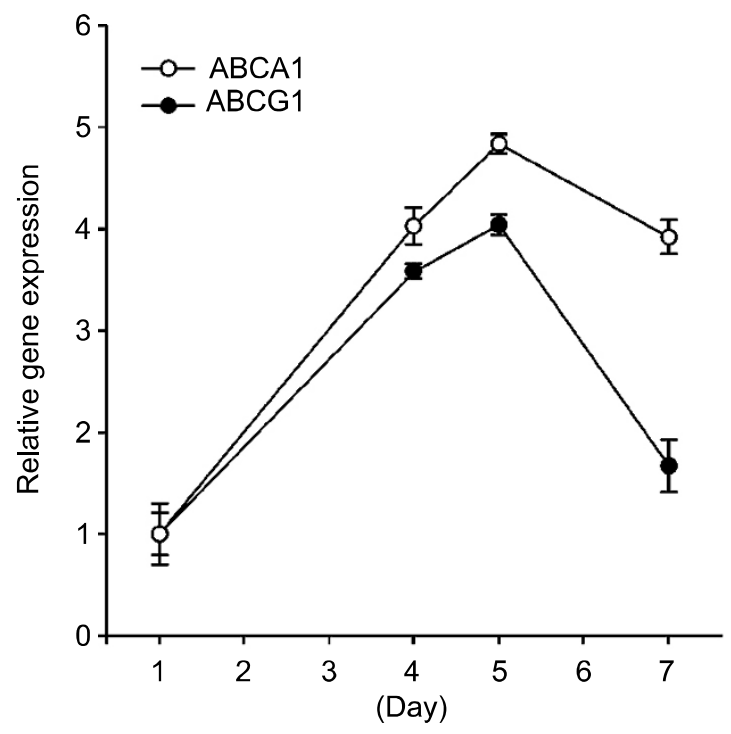

C

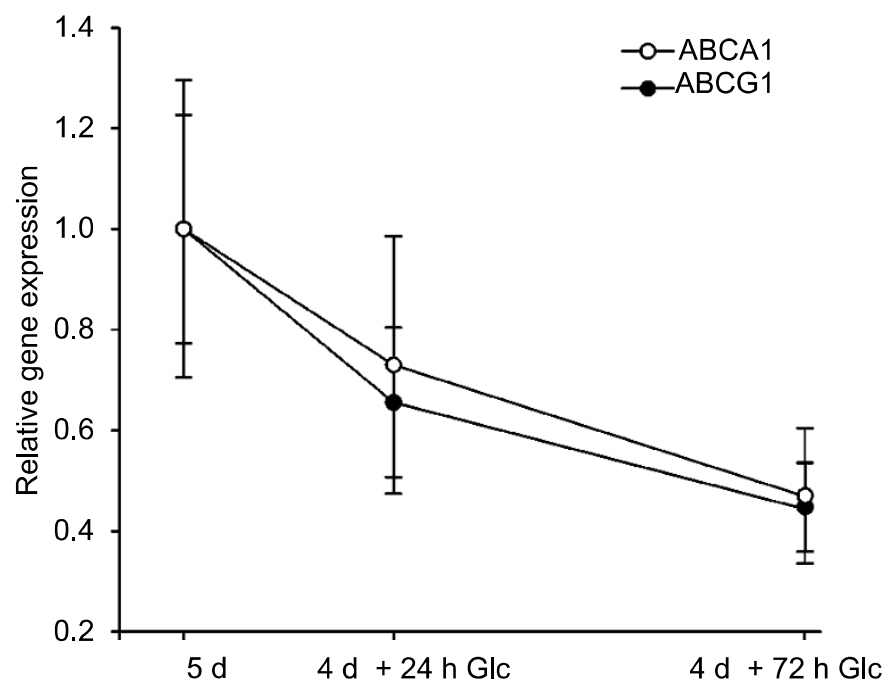

\section{Results}

Macrophage expression profile of all 47 human ATP-binding cassette transporter genes following stimulation with bioactive metabolic factors

TaqMan low-density arrays (TLDA) for real-time quantitative mRNA analysis were performed from three independent stimulation experiments with cells from independent blood donors. Monocytes were predifferentiated to macrophages for 4 days and were further incubated with different metabolic stimuli for $24 \mathrm{~h}$. The effects of insulin, palmitic acid, linoleic acid, 9-cis-retinoic acid, the LXR-ligand
B

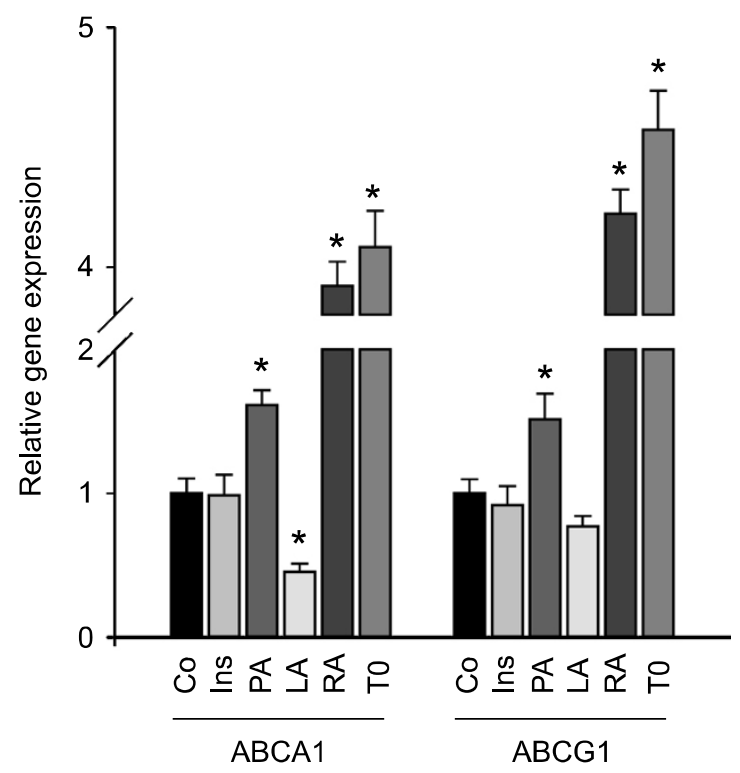

Figure 1. $A B C A 1$ and $A B C G 1$ mRNA levels in human monocyte-derived macrophages as determined by Taqman real-time qRT-PCR. (A) Time kinetic experiments with normal human blood donor macrophages incubated in the presence of M-CSF for the indicated time points. (B) mRNA levels of ABCA1 and ABCG1 after 4 days predifferentiation and further $24 \mathrm{~h}$ incubation in the presence of insulin (Ins), palmitic acid (PA), linoleic acid (LA), 9-cis-retinoic acid (RA) and T0901317 (T0). (C) mRNA levels of ABCA1 and ABCG1 of 5 day differentiated macrophages and macrophage cells predifferentiated for 4 days after further incubation in the presence of high-dose glucose (GIc) for $24 \mathrm{~h}(4 \mathrm{~d}$ $+24 \mathrm{~h} \mathrm{Glc})$ and $72 \mathrm{~h}(4 \mathrm{~d}+72 \mathrm{~h} \mathrm{Glc})$, respectively. Data are presented as mean \pm s.e.m of three independent stimulations. Significance was calculated using Student's t-test, ${ }^{*} P<0.05$. 
T0901317, and glucose were analyzed in comparison to untreated, age-matched control samples. The TLDA results are displayed as a combination of dots and a color code for high (red), medium (yellow), low (blue) and very low (white) expression, which are based on individually calculated $\triangle \mathrm{Ct}$ values [Ct (target gene)-Ct (18S rRNA)] (Table 1). For this analysis, the range between the lowest $\triangle \mathrm{Ct}$ value and a $\triangle \mathrm{Ct}$ of 25 was divided linearly into three groups for high, medium and low expressions. Genes with a $\triangle \mathrm{Ct}$ above 25 cycles were defined as absent. For external calibration, ABC-transporter mRNA levels were also measured in a total RNA pool mixed from several human tissues (universal calibrator). When analyzing the relative expression in 5 day cultured macrophages, we detected transcripts for 31 ABC-transporters, with low to medium levels (Table 1). In concordance with earlier data from our group, 4 day M-CSF predifferentiation and further $24 \mathrm{~h}$ incubation with 9-cis-retinoic acid and T0901317 induced mRNA levels of ABCA1, ABCA2, ABCA7, ABCC2, $A B C D 1, A B C D 4$, and $A B C G 1$. Interestingly, addition of the metabolic compounds insulin, palmitic acid, linoleic acid, and glucose to the medium showed a prominent effect on the expression of both $A B C A 1$ and $A B C G 1$. Thus, palmitic acid induced $A B C A 1 / A B C G 1$ expression while glucose reduced both transcript levels. In addition, ABCA3 and $A B C B 6$ were also differentially regulated with the above mentioned stimuli.

\section{Time-dependent mRNA expression of ABCA1 and ABCG1 following stimulation with bioactive metabolic factors}

Based on the TLDA data and their known role in the development of atherosclerosis, we focused on ABCA1 and ABCG1 for a detailed single sample Taqman analysis of macrophage differentiation and metabolic stimulation. In a time series experiment, monocyte-derived macrophages were cultured in M-CSF-containing medium for 1 day (1 d), 4 days (4 d), 5 days $(5 \mathrm{~d})$, and 7 days $(7 \mathrm{~d})$. ABCA1 and ABCG1 were not expressed in human monocytes (data not shown) and the quantification during macrophage differentiation showed a steep increase until day 5 with a decrease in expression until day 7 (Figure 1A). This indicated that our culture system with stimulation on day 4 resulted in matured macrophages with high $A B C A 1$ and $A B C G 1$ levels. Our single assay analysis fully confirmed the TLDA results and we were able to measure a significant increase of ABCA1 and ABCG1 by palmitic acid, whereas linoleic acid significantly suppressed ABCA1 transcript levels and showed a tendency for reduced expression of $A B C G 1$. The positive controls 9-cis-retinoic acid and T0901317 strongly increased ABCA1 and ABCG1 mRNA levels (Figure 1B). Since our TLDA analysis indicated only a moderate effect of high glucose levels on both genes, a time kinetic experiment was performed. As depicted in Figure 1C, prolonged incubation in the presence of glucose caused only moderate expression changes but continously decreased $A B C A 1$ and $A B C G 1$ transcript levels.

\section{Protein expression of ABCA1 and ABCG1 following stimulation with bioactive metabolic factors}

To recapitulate our interesting mRNA findings on the protein level, we performed western blot analysis with independent macrophage cell extracts.

A

C

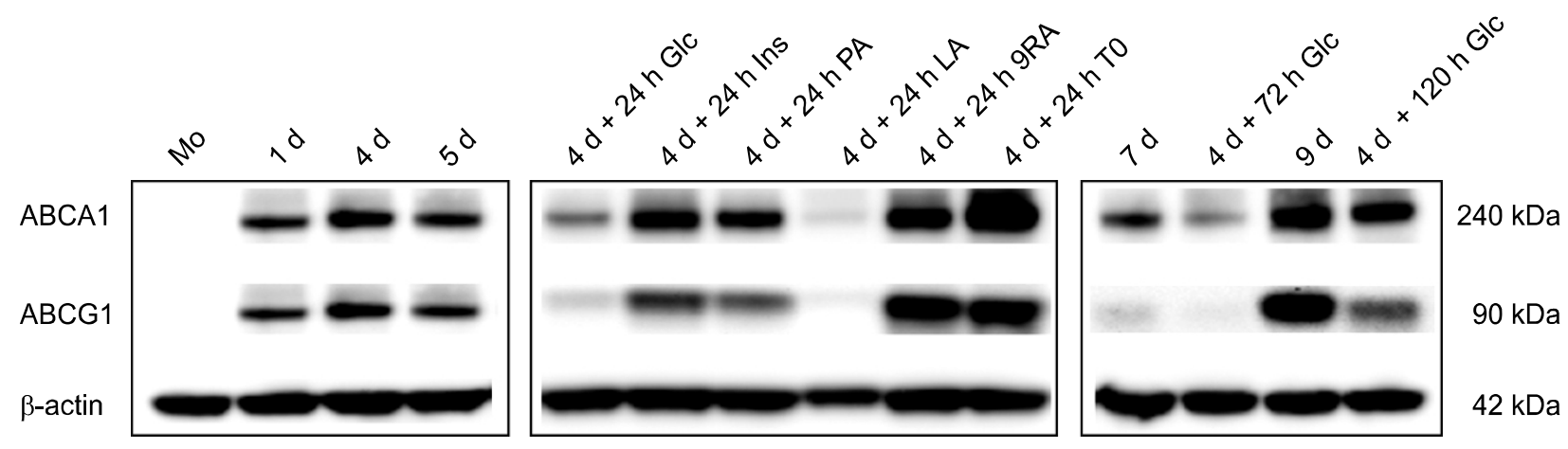

Figure 2. $A B C A 1$ and $A B C G 1$ protein levels in human monocyte-derived macrophages determined by Western blot analysis. (A) Time kinetic experiments with normal human blood donor macrophages incubated in the presence of M-CSF for the indicated time points. (B) Protein levels of ABCA1 and ABCG1 in 4 days predifferentiated macrophages after incubation in the presence of glucose (Glc), insulin (Ins), palmitic acid (PA), linoleic acid (LA), 9-cis-retinoic acid (RA) and T0901317 (T0) for $24 \mathrm{~h}$. (C) Protein levels of ABCA1 and ABCG1 in $7 \mathrm{~d}$ and $9 \mathrm{~d}$ control incubated macrophages or cells after incubation in the presence of high-dose glucose for $72(4 \mathrm{~d}+72 \mathrm{~h} \mathrm{Glc}$ ) and $120 \mathrm{~h}(4$ days $+120 \mathrm{~h} \mathrm{Glc})$, respectively. 
$A B C A 1$ and $A B C G 1$ protein levels strongly increased during macrophage differentiation until day 5 (Figure 2A). Incubation of $4 \mathrm{~d}$ predifferentiated macrophages with insulin and palmitic acid increased the protein content, whereas linoleic acid markedly suppressed the amount of both proteins during a $24 \mathrm{~h}$ culture phase (Figure 2B). We also performed long term glucose culture experiments (Figure 2C), partially reflecting the mRNA data (Figure 1C). Macrophages treated with high glucose for $72 \mathrm{~h}$ and $120 \mathrm{~h}$ displayed a markedly decreased ABCA1 and $A B C G 1$ expression profiles, when compared to control incubated cells (Figure $2 \mathrm{C}$ ).

\section{Discussion}

In this study, we have shown that different metabolic factors elevated in the metabolic syndrome and type 2 diabetes affect the expression of $A B C A 1$ and $A B C G 1$ in primary human monocytederived macrophages and may thus contribute to the development of atherosclerotic lesions in these conditions. In contrast, most of the other $A B C$ transporters were not influenced by metabolic stimuli and therefore may not be involved in the pathogenesis of CVD. We showed that linoleic acid, an $\omega 6$ unsaturated fatty acid, suppressed $A B C A 1$ and $A B C G 1$ transcripts and protein levels in human macrophages. In contrast, the saturated fatty acid palmitic acid had the opposite effect. Our data with primary human macrophages are in line with previous experiments employing murine and human macrophage cell lines (Uehara et al., 2002, 2007). Interestingly it has been reported, that in monkeys a diet rich in saturated palmitic acid leads to higher total cholesterol and HDL levels compared with a diet rich in polyunsaturated linoleic acid (Rudel et al., 1990; Khosla and Hayes, 1992). The reduction in total cholesterol was mainly due to a reduction of $\mathrm{HDL}$ cholesterol. This resulted in a higher total cholesterol/ HDL (TC/HDL) ratio, which has been shown to be highly correlated with ischemic heart disease (Lewington et al., 2007). As our findings show that palmitic acid has a stimulating effect on $A B C A 1$ and $A B C G 1$ expression it is tempting to speculate that dietary intake of palmitic acid could improve TC/HDL ratio. However, Hodson et al. (2001) even found a slightly decreased TC/HDL ratio in young adults eating self-selected diets either rich in saturated or polyunsaturated fatty acids. This may be due to the fact that the free-selected diet considerably differed in their fatty acid compositions compared to standardized diets under laboratory conditions, i.e. the PUFA diet contained almost as much palmitic acid as the SFA diet.

Importantly, our incubation experiments with high levels of glucose identified a clear reduction of both $A B C A 1$ and $A B C G 1$ mRNA and protein expression. These results are consistent with findings in experimental type 2 diabetic mice, which showed diminished ABCG1 expression in peritoneal macrophages (Mauldin et al., 2006). Supporting evidence for our data in humans comes from a recent study showing a negative correlation between blood glucose concentration and leukocyte ABCA1 levels (Albrecht et al., 2004). In addition to glucose, advanced glycated end products (AGEs), which are generated during persistent hyperglycemia, could be involved in destabilizing the ABCA1 protein (Passarelli et al., 2005).

Our data on the synchronous regulation of $A B C A 1$ and $A B C G 1$ by metabolic factors clearly point out a synergistic effect of dysregulated $A B C A 1$ and $A B C G 1$ in the pathophysiology of atheroslerosis. This hypothesis is corroborated by studies with $A b c a 1^{-1-}$ and $A b c g 1^{-1-}$ knockout mice. Thus, LDL-receptor deficient mice transplanted with $\mathrm{Abca} 1^{-1-}$ Abcg $1^{-/ 2}$ double-knockout bone marrow displayed increased foam cell accumulation, inflammatory cell infiltration and apoptosis resulting in enhanced atherosclerosis (Yvan-Charvet et al., 2007). In addition, reverse cholesterol transport, the primary mechanism by which HDL protects against atherosclerosis (Cuchel and Rader, 2006), is more severely impaired in ABCA1 and ABCG1 double-knockdown macrophages, compared to single knockdown models (Wang et al., 2007). In summary, the studies described here provide a first mechanistic link between blood lipid and glucose levels and macrophage reverse cholesterol transport function.

\section{Methods}

\section{Monocyte isolation and differentiation}

Human peripheral blood monocytes were obtained from three healthy blood donors by leukapheresis and counterflow elutriation. Cells were cultured $\left(5 \% \mathrm{CO}_{2}\right.$ in air, $\left.37^{\circ} \mathrm{C}\right)$ on plastic petridishes in macrophage SFM (Gibco, Graud Island, NY) containing $25 \mathrm{ng} / \mathrm{ml}$ of recombinant human macrophage colony stimulating factor (M-CSF) (R\&D systems, Minneapolis, MN). The cells were allowed to differentiate into macrophages for different indicated time points ( 1 to 9 days). Then, the M-CSF containing medium was replenished and the stimuli were added as described below.

\section{Macrophage stimulations}

Four days predifferentiated macrophages were cultured in the presence of $25 \mathrm{mM}$ glucose, $100 \mathrm{nM}$ insulin, $100 \mu \mathrm{M}$ 
linoleic acid, $100 \mu \mathrm{M}$ palmitic acid, $10 \mu \mathrm{M}$ 9-cis retinoic acid, and $10 \mu \mathrm{M}$ T0901317 or control medium for 24 to 120 h. For RNA isolation, cells were scraped in RLT buffer (Qiagen, Valencia, CA) and stored at $-80^{\circ} \mathrm{C}$ until use. For protein isolation, cells were washed with PBS containing a protease inhibitor cocktail (Calbiochem, La Jolla, CA). After centrifugation, cell pellets were lysed in RIPA buffer (Pierce, Rockford, IL).

\section{RNA isolation and reverse transcription}

Total RNA was extracted using the RNeasy Protect Midi Kit (Qiagen). Purity and integrity of the RNA was assessed on the Agilent 2100 bioanalyzer with the RNA 6000 Nano LabChip reagent set (Agilent Technologies, Palo Alto, CA). The RNA was quantified spectrophotometrically and then stored at $-80^{\circ} \mathrm{C}$. cDNAs were generated using the Reverse Transcription System (Promega, Madison, WI).

\section{Analysis of human ATP-binding cassette transporter gene expression by real-time quantitative RT-PCR}

$2 \mu \mathrm{l}$ of single-stranded cDNA (equivalent to $100 \mathrm{ng}$ of total RNA) were mixed with $48 \mu$ l of nuclease-free water and 50 $\mu$ of TaqMan Universal PCR Master Mix (Applied Biosystems, Fosfer City, CA). TaqMan low density arrays (TLDA, Applied Biosystems), which were previously developed by our group (Langmann et al., 2006), were loaded with 100 $\mu l$ of a sample-specific PCR mixture, centrifuged twice for $1 \mathrm{~min}$ at $280 \mathrm{~g}$ and sealed. Real-time quantitative RT-PCR was performed with an ABI Prism 7900 HT Sequence Detection System (Applied Biosystems). The thermal cycling conditions were as follows: $2 \mathrm{~min}$ at $50^{\circ} \mathrm{C}$ hold, 10 min at $95^{\circ} \mathrm{C}$ hold, followed by 40 cycles of $15 \mathrm{~s}$ at $97^{\circ} \mathrm{C}$ melt and $1 \mathrm{~min}$ at $60^{\circ} \mathrm{C}$ anneal/extension. Measurements were carried out in duplicates. Results were analyzed with the $A B I$ sequence detector software (version 2.2) using the $\Delta \Delta \mathrm{Ct}$ method for relative quantitation. Alle experiments were repeated three times.

\section{Western blot analysis}

The protein concentration of cell lysates was determined photometrically using the BCA Protein Assay kit (Pierce) and the concentration was adjusted to $1 \mu \mathrm{g} / \mathrm{L}$ by methanol/ chloroform precipitation followed by resuspension in $1 \times$ sample buffer containing DTT. Proteins were heat-denatured and equal amounts $(15 \mu \mathrm{g})$ were loaded and separated on NuPage SDS gels (Invitrogen, Gaithersburg, $\mathrm{MD}$ ). After transfer, nitrocelluose membranes were blocked with milk powder (1\%) for $1 \mathrm{~h}$ and were subsequently incubated with primary antibodies for $1 \mathrm{~h}$. For the detection of $A B C A 1$ and $A B C G 1$, a monoclonal anti-human ABCA1 antibody (1:1000) (Abcam, Cambridge, MA) and a polyclonal anti-human ABCG1 antibody (1:1000) (Abcam) were used, respectively. A monoclonal antibody against $\beta$-actin $(1: 40000)$ (Abcam) served as a loading control. The primary antibodies were detected with a HRP-labled anti IgG secondary antibody (1:20000) (Invitrogen) in a chemoluminescence reaction. All immunoblots were repeated at least three times and representative experiments are shown.

\section{Acknowledgments}

We thank Dr. Alfred Boettcher for helpful discussions on protein data. This work was supported by Applied Biosystems.

\section{References}

Albrecht C, Simon-Vermot I, Elliott JI, Higgins CF, Johnston DG, Valabhji J. Leukocyte ABCA1 gene expression is associated with fasting glucose concentration in normoglycemic men. Metabolism 2004;53:17-21

Cuchel M, Rader DJ. Macrophage reverse cholesterol transport: key to the regression of atherosclerosis? Circulation 2006;113:2548-55

Fu X, Menke JG, Chen Y, Zhou G, MacNaul KL, Wright SD, Sparrow CP, Lund EG. 27-hydroxycholesterol is an endogenous ligand for liver $X$ receptor in cholesterol-loaded cells. J Biol Chem 2001;276:38378-87

Hodson L, Skeaff CM, Chisholm WA. The effect of replacing dietary saturated fat with polyunsaturated or monounsaturated fat on plasma lipids in free-living young adults. Eur J Clin Nutr 2001;55:908-15

Kennedy MA, Venkateswaran A, Tarr PT, Xenarios I, Kudoh J, Shimizu N, Edwards PA. Characterization of the human $A B C G 1$ gene: liver $X$ receptor activates an internal promoter that produces a novel transcript encoding an alternative form of the protein. J Biol Chem 2001;276:39438-47

Khosla P, Hayes KC. Comparison between the effects of dietary saturated (16:0), monounsaturated (18:1), and polyunsaturated (18:2) fatty acids on plasma lipoprotein metabolism in cebus and rhesus monkeys fed cholesterolfree diets. Am J Clin Nutr 1992;55:51-62

Klucken J, Buchler C, Orso E, Kaminski WE, PorschOzcurumez M, Liebisch G, Kapinsky M, Diederich W, Drobnik W, Dean M, Allikmets R, Schmitz G. ABCG1 (ABC8), the human homolog of the Drosophila white gene, is a regulator of macrophage cholesterol and phospholipid transport. Proc Natl Acad Sci USA 2000;97:817-22

Langmann T, Klucken J, Reil M, Liebisch G, Luciani MF, Chimini G, Kaminski WE, Schmitz G. Molecular cloning of the human ATP-binding cassette transporter 1 (hABC1): evidence for sterol-dependent regulation in macrophages. Biochem Biophys Res Commun 1999;257:29-33

Langmann T, Mauerer R, Schmitz G. Human ATP-binding cassette transporter TaqMan low-density array: analysis of macrophage differentiation and foam cell formation. Clin Chem 2006;52:310-3

Lee JS, Pinnamaneni SK, Eo SJ, Cho IH, Pyo JH, Kim CK, Sinclair AJ, Febbraio MA, Watt MJ. Saturated, but not $n-6$ polyunsaturated, fatty acids induce insulin resistance: role of intramuscular accumulation of lipid metabolites. J Appl Physiol 2006;100:1467-74

Lewington S, Whitlock G, Clarke R, Sherliker P, Emberson 
J, Halsey J, Qizilbash N, Peto R, Collins R. Blood cholesterol and vascular mortality by age, sex, and blood pressure: a meta-analysis of individual data from 61 prospective studies with 55,000 vascular deaths. Lancet 2007;370:1829-39

Mauldin JP, Srinivasan S, Mulya A, Gebre A, Parks JS, Daugherty A, Hedrick CC. Reduction in ABCG1 in Type 2 diabetic mice increases macrophage foam cell formation. $J$ Biol Chem 2006;281:21216-24

Passarelli M, Tang C, McDonald TO, O'Brien KD, Gerrity RG, Heinecke JW, Oram JF. Advanced glycation end product precursors impair ABCA1-dependent cholesterol removal from cells. Diabetes 2005;54:2198-205

Rudel LL, Haines JL, Sawyer JK. Effects on plasma lipoproteins of monounsaturated, saturated, and polyunsaturated fatty acids in the diet of African green monkeys. J Lipid Res 1990;31:1873-82

Savage DB, Petersen KF, Shulman GI. Disordered lipid metabolism and the pathogenesis of insulin resistance. Physiol Rev 2007;87:507-20

sSchmitz G, Langmann T. Transcriptional regulatory networks in lipid metabolism control ABCA1 expression. Biochim Biophys Acta 2005;1735:1-19
Uehara Y, Engel T, Li Z, Goepfert C, Rust S, Zhou X, Langer C, Schachtrup C, Wiekowski J, Lorkowski S, Assmann G, von Eckardstein A. Polyunsaturated fatty acids and acetoacetate downregulate the expression of the ATP-binding cassette transporter A1. Diabetes 2002;51:2922-8

Uehara Y, Miura S, von Eckardstein A, Abe S, Fujii A, Matsuo Y, Rust S, Lorkowski S, Assmann G, Yamada T, Saku K. Unsaturated fatty acids suppress the expression of the ATP-binding cassette transporter G1 (ABCG1) and ABCA1 genes via an $L X R / R X R$ responsive element. Atherosclerosis 2007;191:11-21

Venkateswaran A, Laffitte BA, Joseph SB, Mak PA, Wilpitz DC, Edwards PA, Tontonoz P. Control of cellular cholesterol efflux by the nuclear oxysterol receptor LXR alpha. Proc Natl Acad Sci USA 2000;97:12097-102

Wang X, Collins HL, Ranalletta M, Fuki IV, Billheimer JT, Rothblat GH, Tall AR, Rader DJ. Macrophage ABCA1 and ABCG1, but not SR-BI, promote macrophage reverse cholesterol transport in vivo. J Clin Invest 2007;117:2216-24

Yvan-Charvet L, Ranalletta M, Wang N, Han S, Terasaka N, Li R, Welch C, Tall AR. Combined deficiency of ABCA1 and ABCG1 promotes foam cell accumulation and accelerates atherosclerosis in mice. J Clin Invest 2007;117:3900-8 